\title{
Singapore SPICE: sedation practices in intensive care evaluation in Singapore - a prospective cohort study of the public healthcare system
}

\author{
Shin Yi $\underline{N g}^{1}$, MBBS, MMed, Jason Phua ${ }^{2}$, MRCP, Yu Lin Wong $^{3}$, MBBS, FANZCA, Ganesh Kalyanasundaram ${ }^{4}$, MBBS, MRCP, \\ Amartya Mukhopadhyay ${ }^{2}$, MBBS, MRCP, Danny Lim ${ }^{5}$, MBBS, FAnZCA, Naville Chia ${ }^{6}$, MBBS, MMed, \\ Benjamin Choon Heng $\underline{H o}^{7}$, MBBS, MRCP, Michael J $\underline{\text { Bailey }}{ }^{8}$, PhD, MSc, Yahya Shehabi ${ }^{9}$, PhD, FCICM, Lian Kah $\underline{T i}^{10}$, MBBS, MMed
}

INTRODUCTION A study was conducted to describe the sedation practices of intensive care units (ICUs) in Singapore in terms of drug use, sedation depth and the incidence of delirium in both early ( $<48$ hours) and late ( $>48$ hours) periods of ICU admission.

METHODS A prospective multicentre cohort study was conducted on patients who were expected to be sedated and ventilated for over 24 hours in seven ICUs (surgical ICU, $n=4$; medical ICU, $n=3$ ) of four major public hospitals in Singapore. Patients were followed up to 28 days or until ICU discharge, with four-hourly sedation monitoring and daily delirium assessment by trained nurses. The Richmond Agitation and Sedation Scale (RASS) and Confusion Assessment Method for the Intensive Care Unit (CAM-ICU) were used.

RESULTS We enrolled 198 patients over a five-month period. The mean Acute Physiology and Chronic Health Evaluation (APACHE) II score was $25.3 \pm 9.2$, and $90.9 \%$ were emergency hospital admissions. Patients were followed up for 1,417 ICU patient days, of which 396 days were in the early period and 1,021 days were in the late period. 7,354 RASS assessments were performed. Propofol and fentanyl were the sedative agents of choice in the early and late periods, respectively. Patients were mostly in the light sedation range, especially in the late period. At least one episode of delirium was seen in $23.7 \%$ of patients.

CONCLUSION Sedation practices in Singapore ICUs are characterised by light sedation depth and low incidence of delirium, possibly due to the drugs used.

Keywords: delirium, intensive care unit, sedation

\section{INTRODUCTION}

Delirium is a common complication associated with worse long-term outcomes in critically ill patients. ${ }^{(1,2)}$ Causes of delirium are multifactorial and may be associated with sedation practices. ${ }^{(3,4)}$ Although there are updated guidelines for sedation and analgesia in the intensive care unit (ICU), ${ }^{(5)}$ compliance has typically been low. ${ }^{(6)}$ The Australian and New Zealand Sedation Practice in Intensive Care Evaluation (ANZ SPICE) study found that early sedation depth independently predicts delayed extubation and increased mortality in the ICU, (4) and early goal-directed sedation may be a feasible intervention to improve outcomes. ${ }^{(7)}$

To our knowledge, data on sedation and delirium in critically ill patients in Singapore is limited. A point prevalence survey done in 2010 showed that sedation was administered in $25.8 \%$ of patients; in $75 \%$ of patients, sedation scales were used. However, sedation protocols were only used in $20.8 \%$ of patients. No delirium screening was performed. ${ }^{(8)}$ In view of this, we conducted a prospective multicentre cohort study to comprehensively describe the sedation practices of Singapore ICUs in terms of drug use, sedation depth and incidence of delirium in both the early and late periods of ICU admission.

\section{METHODS}

All surgical and medical adult ICUs in the Singapore public healthcare system (five hospitals: surgical ICU, $\mathrm{n}=5$; medical ICU, $\mathrm{n}=5$ ) were invited to participate in the study. Seven ICUs (surgical $\mathrm{ICU}, \mathrm{n}=4$; medical ICU, $\mathrm{n}=3$ ) from four hospitals eventually agreed to participate. The ANZ SPICE protocol ${ }^{(4)}$ was used. All study centres follow a closed model of care led by attending intensivists. There was an on-duty specialist registrar at all times and the nursing ratio was 1:1 to $1: 2$ in all ICUs. Ethics approval was obtained at all the individual participating centres. The requirement for informed consent was waived due to the observational nature of the study.

All consecutively sedated, intubated and mechanically ventilated adult ICU patients who were expected to remain intubated for another day were included. Patients with suspected or proven dementia, neurological impairment, psychiatric illnesses or those who were unable to communicate with the

\footnotetext{
${ }^{1}$ Department of Surgical Intensive Care, Division of Anaesthesiology and Perioperative Medicine, Singapore General Hospital, ${ }^{2} \mathrm{FAST}$ and Chronic Programmes, Alexandra Hospital, National University Hospital, National University Health System, ${ }^{3}$ Department of Anaesthesiology, Intensive Care and Pain Medicine, Tan Tock Seng Hospital, ${ }^{4}$ Department of Medicine, Sengkang General Hospital, ${ }^{5}$ Department of Anaesthesia, National University Hospital, National University Health System, ${ }^{6}$ Department of Anaesthesia, Khoo Teck Puat Hospital, ${ }^{7}$ Department of Respiratory and Critical Care Medicine, Tan Tock Seng Hospital, Singapore, ${ }^{8}$ Australian and New Zealand Intensive Care Research Centre, School of Public Health and Preventive Medicine, ${ }^{9}$ School of Clinical Sciences, Faculty of Medicine, Nursing and Health Sciences, Monash University, Melbourne, Australia, ${ }^{10}$ Department of Anaesthesia, NUS Yong Loo Lin School of Medicine, National University Health System, Singapore

Correspondence: Dr Ng Shin Yi, Senior Consultant, Department of Surgical Intensive Care, Division of Anaesthesiology and Perioperative Medicine, Singapore General Hospital, Outram Road, Singapore 169608.ng.shin.yi@singhealth.com.sg
} 
investigators were excluded. To avoid over-representation by any one centre, no centre recruited more than 30 patients.

Prior to the commencement of the study, the investigators, research staff and ICU nurses were trained in the ANZ SPICE protocol, ${ }^{(4)}$ including assessment of patients using the Richmond Agitation and Sedation Scale (RASS) ${ }^{(9)}$ and Confusion Assessment Method for the Intensive Care Unit (CAM-ICU). ${ }^{(10)}$ The local languages (i.e. English, Mandarin, Malay, Tamil and local dialects) were used to communicate with the patients. We used the ANZ SPICE study protocol ${ }^{(4)}$ with a streamlined standardised case report form for data entry. A trained research coordinator collected the data from electronic and paper clinical notes.

Upon enrolment into the study, relevant demographic data, including age, gender, weight, Acute Physiology and Chronic Health Evaluation (APACHE) II ${ }^{(11)}$ scores and admission sources, was collected. RASS and pain scores were assessed by trained nurses at baseline and every four hours subsequently. Patients' sedation level was recorded according to the RASS as light sedation (score range -2 to +1 ), deeply sedated (score range -3 to -5 ) and agitated (score $\geq 2$ ). Daily CAM-ICU assessments were only performed for lightly sedated patients. Patients were diagnosed with delirium if their CAM-ICU result was positive. For patients who were able to communicate, a visual analogue scale score $>3$ was used for pain assessment. For those who were unable to communicate, Critical Care Pain Observation Tool descriptors ${ }^{(12)}$ were used.

The first 48 hours of ICU admission were considered as the early period. A patient was considered to have early deep sedation if a RASS score of -3 to -5 was recorded during this period. The period after the first 48 hours of ICU admission was considered as the late period.

Data on all cumulative sedative medication infusions and doses were collected. Daily sedation cessations and indications were noted. Adjunct therapies, such as the use of physical restraints, renal replacement therapy and vasopressors, were recorded as well. Patients were followed up until discharge from hospital or for up to 28 days. We recorded outcomes of development of delirium, tracheostomy performed, ventilation duration, ICU mortality and length of stay, and hospital mortality and length of stay.

Statistical analysis was performed using SPSS Statistics version 17.0 (SPSS Inc, Chicago, IL, USA). Comparisons of categorical data and proportion were performed using chi-square or Fisher's exact test, as appropriate. Parametric continuous variables were compared using Student's $t$-test. Non-parametric variables were compared using Wilcoxon rank-sum test. All tests were two-sided and $p<0.05$ was considered to be statistically significant. Data was presented as mean \pm standard deviation or median (interquartile range [IQR]), as appropriate. Missing data was handled with multiple imputations.

\section{RESULTS}

We enrolled 198 patients over a period of five months in 2012. A majority of the patients were admitted from the ward $(n=79$, $39.9 \%)$, followed by the emergency department $(n=56,28.3 \%)$ and emergency operating rooms $(n=45,22.7 \%)$. Together,
Table I. Patient demographics $(\mathbf{n}=198)$.

\begin{tabular}{|ll|}
\hline Variable & No. $(\%) /$ mean \pm SD \\
\hline Age (yr) & $62.3 \pm 16.9$ \\
\hline Male gender & $109(55.1)$ \\
\hline Weight $(\mathbf{k g})$ & $62.3 \pm 15.1$ \\
\hline APACHE II score & $25 \pm 9$ \\
\hline Cause of hospital admission & \\
\hline Ward & $79(39.9)$ \\
\hline Emergency department visit & $56(28.3)$ \\
\hline Emergency operation & $45(22.7)$ \\
\hline Elective operation & $10(5.1)$ \\
\hline Intensive care unit transfer & $7(3.5)$ \\
\hline Interhospital transfer & $1(0.5)$ \\
\hline
\end{tabular}

APACHE: Acute Physiology and Chronic Health Evaluation; SD: standard deviation

Table II. Drug regimens in the early and late periods.

\begin{tabular}{|c|c|c|c|}
\hline \multirow[t]{2}{*}{ Drug } & \multicolumn{3}{|c|}{ No. (\%)/mean \pm SD } \\
\hline & $\begin{array}{l}\text { Total } \\
(n=1,417)\end{array}$ & $\begin{array}{l}\text { Early period } \\
(n=396)\end{array}$ & $\begin{array}{l}\text { Late period } \\
(n=1,021)\end{array}$ \\
\hline \multicolumn{4}{|l|}{ Fentanyl } \\
\hline No. of patient days & $577(40.7)$ & $193(48.7)$ & $384(37.6)$ \\
\hline Dose (mg) & 401,319 & 112,159 & 289,160 \\
\hline Dose/patient day (mg) & $696 \pm 403$ & $581 \pm 451$ & $753 \pm 489$ \\
\hline \multicolumn{4}{|l|}{ Propofol } \\
\hline No. of patient days & $511(36.1)$ & $260(65.7)$ & $251(24.6)$ \\
\hline Dose (mg) & 528,369 & 244,432 & 283,937 \\
\hline Dose/patient day (mg) & $1,034 \pm 726$ & $940 \pm 783$ & $1,131 \pm 872$ \\
\hline \multicolumn{4}{|l|}{ Morphine } \\
\hline No. of patient days & $228(16.1)$ & $76(19.2)$ & $152(14.9)$ \\
\hline Dose (mg) & 5,185 & 1,370 & 3,815 \\
\hline Dose/patient day (mg) & $23 \pm 13$ & $18 \pm 18$ & $25 \pm 15$ \\
\hline \multicolumn{4}{|l|}{ Midazolam } \\
\hline No. of patient days & $162(11.4)$ & $77(19.4)$ & $85(8.3)$ \\
\hline Dose (mg) & 5,542 & 1,972 & 3,570 \\
\hline Dose/patient day (mg) & $34 \pm 32$ & $26 \pm 36$ & $42 \pm 37$ \\
\hline \multicolumn{4}{|l|}{ Dexmedetomidine } \\
\hline No. of patient days & $57(4.0)$ & $17(4.3)$ & $40(3.9)$ \\
\hline Dose (mg) & 166 & 32 & 134 \\
\hline Dose/patient day (mg) & $2.9 \pm 1.8$ & $1.9 \pm 1.3$ & $3.4 \pm 2.1$ \\
\hline Ketamine & $2^{*}$ & & \\
\hline Haloperidol & $1 *$ & & \\
\hline Diazepam & $1 *$ & & \\
\hline
\end{tabular}

${ }^{*}$ No. of patient days. ICU: intensive care unit; SD: standard deviation

this group of patients was considered as emergency hospital admissions ( $n=180,90.9 \%$ ). The baseline demographics of our primary cohort are presented in Table I.

Of the 162 patients who were admitted for more than 48 hours, 49 (30.2\%) patients were lightly sedated during the first 48 hours. All patients recruited were followed until the end of the study. In total, our cohort was followed up for 1,417 ICU patient days, of which 396 patient days were in the early period (first 48 hours) and 1,021 patient days were in the late period (after 48 hours). 


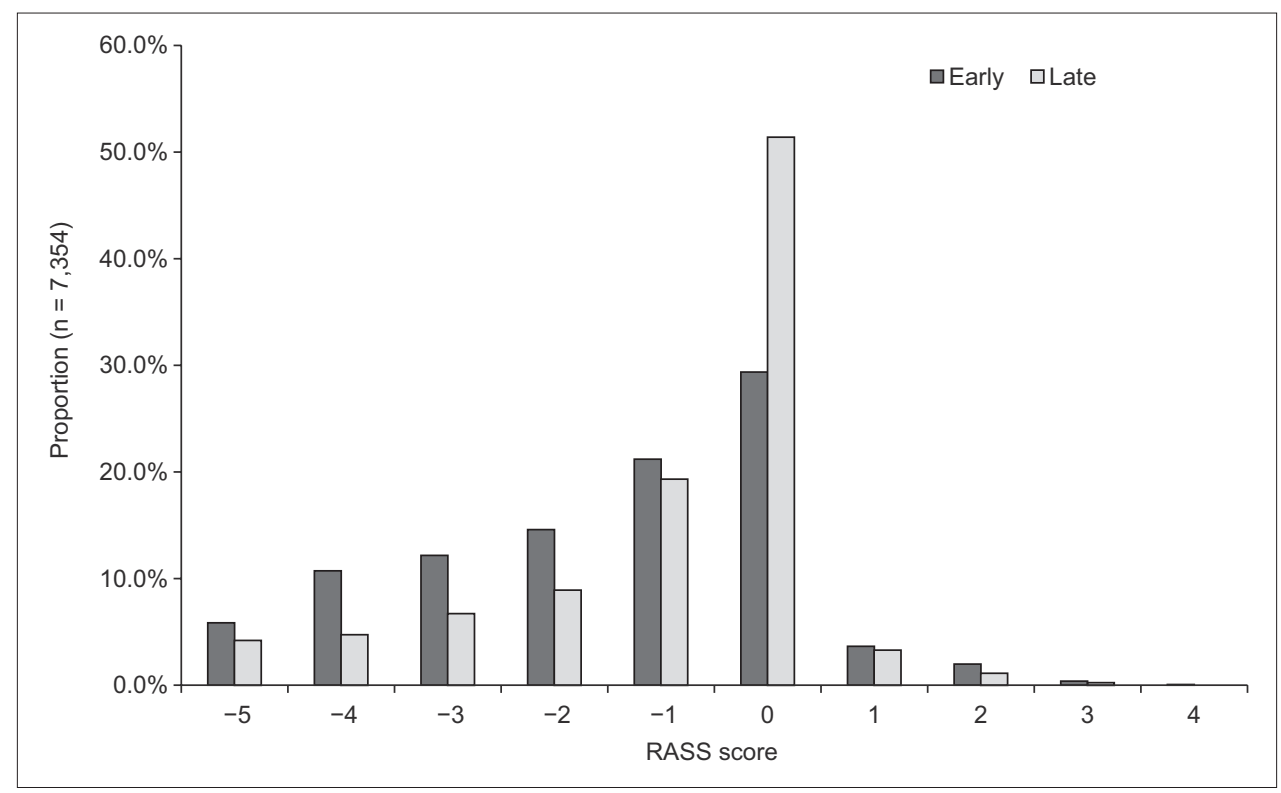

Fig. 1 Bar graph shows the comparative sedation levels, according to RASS assessments, during the early and late periods of ICU admission. ICU: intensive care unit; RASS: Richmond Agitation and Sedation Scale

Table III. Sedation holidays.

\begin{tabular}{|ll|}
\hline Variable & No. (\%) \\
\hline No. of patient days & $1,417(100.0)$ \\
\hline Deliberate cessation & $313(22.1)$ \\
\hline Indication $(\mathbf{n}=\mathbf{3 1 3})$ & \\
\hline Extubation & $174(55.6)$ \\
\hline Deep sedation not required & $22(7.0)$ \\
\hline Routine daily interruption & $13(4.2)$ \\
\hline Other & $110(35.1)$ \\
\hline
\end{tabular}

The overall drug regimen and the drug regimens of the early and late periods are presented in Table II. In the early period, the most commonly used sedation drug was propofol, which was administered for $260(65.7 \%)$ patient days, followed by fentanyl ( $n=193,48.7 \%$ ). In the late period, the reverse trend was observed, with fentanyl being more commonly administered than propofol (fentanyl: $\mathrm{n}=384,37.6 \%$; propofol: $\mathrm{n}=251$, $24.6 \%$ ). While the proportion of patients receiving sedation drugs decreased from the early period to late period, the mean dose of drug per patient day increased. Sedation holidays were practised in about one-fifth (22.1\%) of our patients, for whom extubation was the main indication (55.6\%; Table III).

7,354 RASS assessments were performed on 1,417 ICU patient days. For 2,849 (38.7\%) RASS assessments, the ICU team prescribed a sedation target, which was met most of the time $(82.5 \%)$. Overall, 5,836 (79.4\%) of the sedations performed were in the light sedation range. This occurred more often in the late period when compared to the early period (late period $82.9 \%$; early period $69.8 \%$ ). In contrast, there were more deep sedations in the early period when compared to the late period (early period $28.8 \%$; late period $15.7 \%$ ). The range of sedation levels achieved according to RASS scores over the early and late periods are presented in Fig. 1.

Out of 198 patients, $47(23.7 \%)$ patients had at least one episode of delirium during the ICU stay. CAM-ICU was positive in
$164(11.6 \%)$ patient days, with more occurring in the late period $(\mathrm{n}=137,13.4 \%)$ when compared to the early period $(\mathrm{n}=27$, $6.8 \%$ ). The overall incidence of pain was $8.4 \%$. Data for intensive care sedation, delirium and pain is presented in Table IV.

Patient outcomes are presented in Table V. Overall ICU and hospital mortality were $12.1 \%$ and $24.2 \%$, respectively. The median ICU and hospital lengths of stay were 4 (IQR 3-8) days and 21 (IQR 11-37) days, respectively. The median number of days on the ventilator was 3 (IQR 2-6) days, and 14 (7.1\%) patients received a tracheostomy.

\section{DISCUSSION}

Over a period of five months, we observed the sedation practices for 198 ICU patients in the Singapore public healthcare system. Propofol and fentanyl were the sedative agents of choice in the early and late periods, respectively. Patients were mostly in the light sedation range, and this occurred more commonly in the late period. About one-quarter of our patients had at least one episode of delirium.

Patients in Singapore had a sedation depth profile comparable to that of two previous SPICE studies in Australia/New Zealand (ANZ SPICE) and Malaysia (MY SPICE), ${ }^{(4,13)}$ with a greater proportion of deep sedation scores occurring in the early period when compared to the late period. In Australia, $61.9 \%$ of RASS assessments in the early period were in the deep sedation range when compared to $23.7 \%$ in the late period; ${ }_{i}^{(4)}$ the corresponding proportion was $58 \%$ in the early period and $34 \%$ in the late period for Malaysia, ${ }^{(13)}$ and $28.8 \%$ in the early period and $15.7 \%$ in the late period for Singapore in our study. This is to be expected, as patients may be more acutely ill in the early course of critical illness and require deeper sedation to facilitate critical care interventions.

In general, our patients were more lightly sedated than the other two cohorts. In the early period, $69.8 \%$ of our patients were in the light sedation range compared to $35.5 \%$ and $39.3 \%$ in ANZ 
Table IV. RASS assessments, CAM-ICU and pain during the early and late periods.

\begin{tabular}{|llll|}
\hline \multirow{2}{*}{ Drug } & \multicolumn{3}{c|}{ No. (\%) } \\
\cline { 2 - 4 } & Total & Early period & Late period \\
\hline No. of patient days & $1,417(100.0)$ & $396(100.0)$ & $1,021(100.0)$ \\
\hline With $\geq 1$ assessment of light sedation & $1,075(75.9)$ & $240(60.6)$ & $835(81.8)$ \\
\hline With positive CAM-ICU & $164(11.6)$ & $27(6.8)$ & $137(13.4)$ \\
\hline RASS assessment & $7,354(100.0)$ & $1,863(100.0)$ & $5,491(100.0)$ \\
\hline Had prescribed sedation target & $2,849(38.7)$ & $727(39.0)$ & $2,122(38.6)$ \\
\hline Met target & $2,351(82.5)$ & $566(77.9)$ & $1,785(84.1)$ \\
\hline Could communicate that he was in pain & $269(3.7)$ & $57(3.1)$ & $212(3.9)$ \\
\hline Could not communicate but appeared to be in pain & $351(4.8)$ & $91(4.9)$ & $260(4.7)$ \\
\hline
\end{tabular}

CAM-ICU: Confusion Assessment Method for the Intensive Care Unit; RASS: Richmond Agitation and Sedation Scale

Table V. Patient outcomes $(\mathbf{n}=198)$.

\begin{tabular}{|ll|}
\hline Outcome & No. (\%)/median (IQR) \\
\hline Mortality & $48(24.2)$ \\
\hline Hospital & $24(12.1)$ \\
\hline ICU & $21(11-37)$ \\
\hline LOS (day) & $4(3-8)$ \\
\hline Hospital & $3(2-6)$ \\
\hline ICU & $14(7.1)$ \\
\hline Duration on ventilation (day) & $9(6-15)$ \\
\hline Tracheostomy & $131(66.2)$ \\
\hline Time to tracheostomy (day) & $51(25.8)$ \\
\hline Vasopressor & \\
\hline Renal replacement therapy & \\
\hline
\end{tabular}

ICU: intensive care unit; IQR: interquartile range; LOS: length of stay

SPICE and MY SPICE, respectively. In the late period, $15.7 \%$ of our patients were in the deep sedation range when compared to $23.7 \%-34 \%$ of patients in the other two studies.

CAM-positive delirium occurred at least once in $23.7 \%$ of our patients, which was lower than a range of $44 \%-50.7 \%$ in the other two SPICE studies. In a North American BRAIN-ICU study, ${ }^{(14)} 74 \%$ of critically ill patients developed delirium during the hospital stay. Differences in findings related to the incidence of delirium between these studies may be attributed to the different profiles of their study populations. In addition, the different sedation practices adopted in these hospitals may also have contributed to it. Midazolam was the most common sedative administered in MY SPICE (39.6\% of patient days) and second most common in ANZ SPICE (36.6\% of patient days). In contrast, in our study, propofol and fentanyl were the most common sedative agents administered, with midazolam used only for $11.4 \%$ of patient days. Midazolam, as a benzodiazepine, has been associated with increased incidence and duration of delirium in previous studies. ${ }^{(15,16)}$ The reduced use of midazolam in our practice may have been associated with the lower incidence of delirium observed in our patients.

Due to the limited sample size, while we did characterise a difference in sedation depth between the early and late periods of ICU patient days, we did not analyse the effect of this exposure on clinical outcomes.
To our knowledge, this is the first prospective longitudinal multicentre study of sedation practices in surgical and medical ICUs of the Singapore public healthcare system. The study protocol was detailed and comprehensive, and data collection was supported by an experienced research team. This data is an invaluable baseline for further studies on sedation and delirium in Singapore ICUs.

Due to the research protocol, the completeness of monitoring was very high in our study and may not reflect the practice in a busy ICU. A quality improvement study in Singapore found that compliance to sedation monitoring and delirium screening was $79 \%$ and $36 \%$, respectively. ${ }^{(17)}$ Barriers cited for compliance included difficulty in performing and interpreting delirium screening as well as a lack of appropriate physician response. However, an educational programme in the ICU was able to increase compliance to delirium screening to $61 \%$ at ten months. ${ }^{(17)}$ Hence, future pragmatic research in this context should take into account the effectiveness and sustainability of sedation and delirium monitoring. Interventions in sedation/delirium monitoring and treatment should be aimed at improving clinically relevant outcomes. Changes to practices must be implementable across different critical practices.

The limitations of our study were that we were unable to document the screening process and comprehensively describe the numbers and reasons for exclusion of patients. Selection bias may have resulted, and thus, the external validity and generalisability of our study and its findings was weakened.

Current randomised controlled trials on sedation have not looked at the impact of early sedation depth on outcome. A pilot study suggested that delivery of early goal-directed sedation was feasible, appeared safe, achieved early light sedation, minimised the use of benzodiazepines and propofol, and decreased the need for physical restraints. ${ }^{(7)}$ The completion of SPICE III will provide us with data and insight for translation into practice that is measurable and sustainable. While the development of delirium in ICU patients is associated with worse outcomes, it is not known whether pharmacological treatment of ICU delirium would result in improved outcomes. ${ }^{(18)}$ The MINDS-USA (Modifying the Impact of ICU-Associated Neurological Dysfunction-USA) trial should shed more light on the subject.

The modern practice of ICU sedation is embodied in the concept of eCASH - early Comfort using Analgesia, minimal 
Sedatives and maximal Humane care. ${ }^{(19)}$ Our understanding and practice of sedation and delirium management in the ICU will continue to evolve with the availability of more data.

In conclusion, we found that sedation practices in Singapore's ICUs differ from those of other centres, having less use of benzodiazepines, lighter depth of sedation and less delirium. Data from future studies will further guide our practice.

\section{ACKNOWLEDGEMENT}

This study was supported by a non-restricted grant from Hospira Ltd. The authors had full oversight of the study design, data collection, data analysis and manuscript preparation.

\section{REFERENCES}

1. Ely EW, Shintani A, Truman B, et al. Delirium as a predictor of mortality in mechanically ventilated patients in the intensive care unit. JAMA 2004; 291:1753-62.

2. Ouimet S, Kavanagh BP, Gottfried SB, Skrobik Y. Incidence, risk factors and consequences of ICU delirium. Intensive Care Med 2007; 33:66-73.

3. Skrobik Y, Ahern S, Leblanc M, et al. Protocolized intensive care unit management of analgesia, sedation, and delirium improves analgesia and subsyndromal delirium rates. Anesth Analg 2010; 111:451-63.

4. Shehabi Y, Bellomo R, Reade MC, et al; Sedation Practice in Intensive Care Evaluation (SPICE) Study Investigators; ANZICS Clinical Trials Group. Early intensive care sedation predicts long-term mortality in ventilated critically ill patients. Am J Respir Crit Care Med 2012; 186:724-31.

5. Barr J, Fraser GL, Puntillo K, et al; American College of Critical Care Medicine. Clinical practice guidelines for the management of pain, agitation, and delirium in adult patients in the intensive care unit. Crit Care Med 2013; 41:263-306.

6. Jackson DL, Proudfoot CW, Cann KF, Walsh TS. The incidence of suboptimal sedation in the ICU: a systematic review. Crit Care 2009; 13:R204.

7. Shehabi $Y$, Bellomo R, Reade M, et al; Sedation Practice in Intensive Care Evaluation (SPICE) Study Investigators; Australian and New Zealand Intensive Care Society Clinical Trials Group. Early goal-directed sedation versus standard sedation in mechanically ventilated critically ill patients: a pilot study. Crit Care Med 2013; 41:1983-91.

8. Koh J, Tee A, Phoo JWH, et al. A national point-prevalence survey of the practice of sedation, analgesia, neuromuscular blockade and delirium assessment in adult intensive care units in Singapore. Crit Care Shock 2010; 13:122-31.

9. Sessler CN, Gosnell MS, Grap MJ, et al. The Richmond Agitation-Sedation Scale: validity and reliability in adult intensive care unit patients. Am J Respir Crit Care Med 2002; 166:1338-44.

10. Ely EW, Inouye SK, Bernard GR, et al. Delirium in mechanically ventilated patients: validity and reliability of the confusion assessment method for intensive care unit (CAM-ICU). JAMA 2001; 286:2703-10.

11. Knaus WA, Draper EA, Wagner DP, Zimmerman JE. APACHE II: a severity of disease classification system. Crit Care Med 1985; 13:818-29.

12. Gélinas C, Fillion L, Puntillo KA, Viens C, Fortier M. Validation of the criticalcare pain observation tool in adult patients. Am J Crit Care 2006; 15:420-7.

13. Shehabi Y, Chan L, Kadiman S, et al; Sedation Practice in Intensive Care Evaluation (SPICE) Study Group Investigators. Sedation depth and longterm mortality in mechanically ventilated critically ill adults: a prospective longitudinal multicentre cohort study. Intensive Care Med 2013; 39:910-8.

14. Pandharipande PP, Girard TD, Jackson JC, et al; BRAIN-ICU Study Investigators. Long-term cognitive impairment after critical illness. N Engl J Med 2013; 369:1306-16.

15. Pandharipande PP, Pun BT, Herr DL, et al. Effect of sedation with dexmedetomidine vs lorazepam on acute brain dysfunction in mechanically ventilated patients: the MENDS randomized controlled trial. JAMA 2007; 298:2644-53.

16. Riker RR, Shehabi Y, Bokesch PM, et al; SEDCOM (Safety and Efficacy of Dexmedetomidine Compared with Midazolam) Study Group. Dexmedetomidine vs midazolam for sedation of critically ill patients: a randomized trial. JAMA 2009; 301:489-99.

17. Chen FS, Lieow J, Tang PS, et al. Effectiveness of an APN-led multifaceted education program on bedside nurses' knowledge of delirium and their compliance in recognition of delirium in intensive care units at a tertiary care teaching hospital in Singapore. Presented at the 3rd NUS-NUH International Nursing Conference, 18-20 November 2015, Singapore.

18. Girard T, Pandharipande PP, Carson SS, et al; MIND Trial Investigators. Feasibility, efficacy, and safety of antipsychotics for intensive care unit delirium: the MIND randomized, placebo-controlled trial. Crit Care Med 2010; 38:428-37.

19. Vincent JL, Shehabi Y, Walsh TS, et al. Comfort and patient-centred care without excessive sedation: the eCASH concept. Intensive Care Med 2016; 42:962-71. 